\title{
Connection Between Solar Hemispheric Toroidal Cycles and Geomagnetic Variations
}

\author{
J. Muraközy ${ }^{1}$ iD
}

Received: 31 October 2018 / Accepted: 30 March 2019 / Published online: 19 April 2019

(C) The Author(s) 2019

\begin{abstract}
The solar activity has hemispheric asymmetries. The levels of activity are different on the two hemispheres on intermediate and longer time scales. During four Schwabe cycles the progress of the northern hemispheric activity precedes the southern one, while in the next four cycles the southern cycle takes over the preceding role (Muraközy and Ludmány, Mon. Not. Roy. Astron. Soc. 419, 3624, 2012; Muraközy, Astrophys. J. 826, 145, 2016). The interplanetary magnetic field is formed by the distribution of the solar magnetic fields and the outward-streaming solar wind. The present study intends to show how the solar-hemispheric predominance affects the interplanetary and geophysical magnetic field. The interplanetary and geophysical data sets have been chosen from various sources such as the components of the interplanetary magnetic field $[B]$, cosmic-ray data, Ap, aa, and Dst geomagnetic indices, while the solar-hemispheric asymmetry has been examined by using sunspot data from Greenwich Photoheliographic Results (GPR) and Debrecen Photoheliographic Data (DPD).
\end{abstract}

Keywords Solar cycle · Magnetic fields, interplanetary · Geomagnetic disturbances

\section{Introduction}

The solar-hemispheric-activity differences have been well-known phenomena since several years and from work of many authors (Zolotova and Ponyavin, 2006, 2007; Zhang, Mursula, and Usoskin, 2013), but their origin is not yet clear. There is also a longer sunspot cycle, which consists of two times four Schwabe cycles as is evident in the hemispheric sunspot number and area of the Greenwich and post-Greenwich era during Cycles 12-24 (Muraközy and Ludmány, 2012). This variation cannot be pinpointed clearly in cycles of the pre-Greenwich era, but its existence can be presumed (Muraközy, 2016).

J. Muraközy

murakozy.judit@csfk.mta.hu

1 Debrecen Heliophysical Observatory (DHO), Konkoly Observatory, Research Centre for Astronomy and Earth Sciences, Hungarian Academy of Sciences, P.O. Box 30, 4010 Debrecen, Hungary 
A possible spatial extension of the investigation of the solar-hemispheric asymmetry is the study of the interplanetary field. Wang and Robbrecht (2011) have studied the northsouth displacement of the heliospheric current sheet (HCS) versus the hemispheric activity of the last five cycles. They found that the southward displacement of the HCS follows from Joy's law and the hemispheric asymmetry of the sunspot number. Mursula and Hiltula (2003) and Virtanen and Mursula (2010) also studied the behavior of the HCS and found its so-called "bashful ballerina" structure and found that the high-latitude radial field's absolute value is larger in the southern hemisphere than in the northern one during the observed time spans. Zieger and Mursula (1998) have found north-south asymmetry in the solarwind speed; while Mursula, Hiltula, and Zieger (2002) found a century-scale oscillation of the north-south asymmetry of the streamer belt that is related to the solar magnetic cycles and that the effective latitudinal gradients of the solar-wind speed in the two hemispheres are different. Moreover, these asymmetries are stronger during high solar activity. Rangarajan and Iyemori (1998) studied the hemispherical geomagnetic response to the solar-wind streams and pointed out a hemispheric difference in that phenomenon and showed that the southern hemisphere is more active with $\approx 14$-day periodicity while the northern one has $\approx 28$-day periodicity. Georgieva et al. (2005) have shown that there is a connection between the IMF and solar-hemispheric differential rotation.

There are many investigations of these differences, mainly on shorter time scales, but this article focuses on the north-south differences of the number of sunspot groups and solar flares on longer time scales. In addition, the probable connection with some varying parameters of the IMF, solar-wind, and geomagnetic data are also studied.

\section{Data and Methods}

The present work aims to investigate the hemispheric variation of the solar and the interplanetary magnetic fields during the longest available time span. The problem is that the time spans of these kinds of data are different. Below I describe the investigated data and their time intervals.

The sunspot-group data are taken from the Greenwich Photoheliographic Results (GPR) for the years 1932-1975, while the post-Greenwich data are taken from the Debrecen Photoheliographic Data (DPD: Baranyi, Győri, and Ludmány, 2016; Győri, Baranyi, and Ludmány, 2017). The GPR and DPD contain area as well as position data of all observed sunspot groups. However, while DPD contains such data for all individual sunspots as well, this article takes only the sunspot-group data into account. To describe the hemispheric solar activity, each sunspot group observed during a month has been considered. Each hemispheric solar cycle determined by using sunspot group $\left[N_{\mathrm{G}}\right]$ or solar-flare $\left[N_{\mathrm{F}}\right]$ numbers is described with its center of weight (hereafter $\mathrm{CW}$ ) calculated from the minimum to the next minimum of the activity profiles. It is necessary to deal with each solar cycle as an entity.

The solar-flare data are taken from the Geostationary Operational Environmental Satellites (GOES) data base. The X-ray solar-flare data cover the time span 1975-2017. The monthly hemispheric flare number ( $N_{\mathrm{F}}$ top panel of Figure 1$)$ has been calculated even in those cases when the flare is not associated with an active region. Thus every single flare has been taken into account in a month, as in the case of the hemispheric number of sunspot groups, independently of their intensities. The hemispheric temporal profiles are smoothed with an 11-month window on both panels of Figure 1, but the hemispheric CWs have been calculated by using the unsmoothed monthly data. 


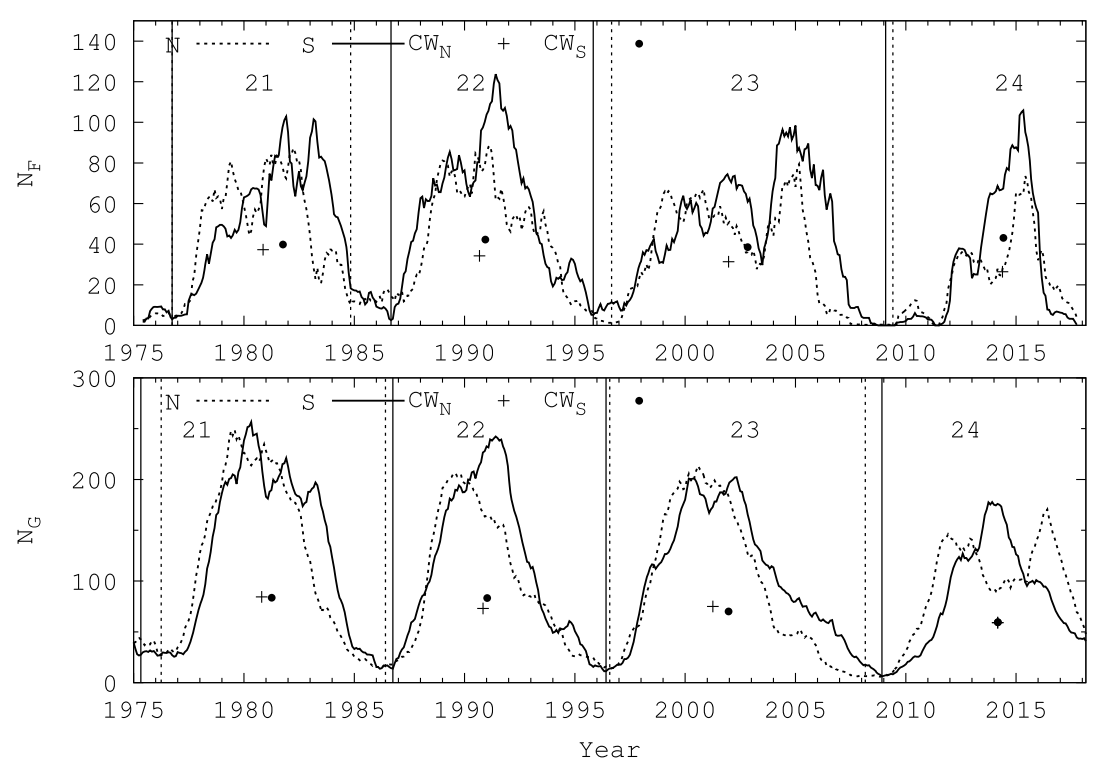

Figure 1 Hemispheric flare number (top panel) and sunspot-group number (bottom panel). The hemispheric-activity profiles are smoothed with an 11-month window. The vertical lines depict the time of the hemispheric minima, while the crosses and dots mark the northern and southern centers of weight of cycle profiles (CWs), respectively.

The interplanetary magnetic-field (IMF) values and solar-wind parameters are taken from the OMNI database, which contains data from several spacecraft measured at $1 \mathrm{AU}$. The planetary A-index [Ap] is taken from the National Centers for Environmental Information (NCEI) while the aa and the Disturbance Storm-Time [Dst] indices are from the International Service of Geomagnetic Indices (ISGI) site and the World Data Center for Geomagnetism, Kyoto.

On the one hand, there are no available long-time IMF data before 1965, the approximate time of the previous change of solar-hemispheric phase lag (Muraközy and Ludmány, 2012). On the other hand, there are no clearly recognizable and hemispheric cycles in the cases of IMF and geomagnetic data. Thus, the possible relationship between them and solarhemispheric phase lag can only be studied by comparing the IMF data as well as the geomagnetic indices and the absolute value of the $\mathrm{N}-\mathrm{S}$ hemispheric difference's monthly variation of the number of sunspot groups $\left[\left|\Delta N_{\mathrm{G}}\right|\right.$, described below], which characterizes the strength of the hemispheric asymmetry. $N_{\mathrm{GN}}$ and $N_{\mathrm{GS}}$ describe the northern and southern hemispheric sunspot-group number, respectively.

$$
\left|\Delta N_{\mathrm{G}}\right|=\left|N_{\mathrm{GN}}\right|-\left|N_{\mathrm{GS}}\right| \text {. }
$$

\section{Results and Discussions}

Figure 1 shows that the shape of hemispheric $N_{\mathrm{F}}$ (top panel of Figure 1) is similar to the shape of the hemispheric $N_{\mathrm{G}}$ (bottom panel of Figure 1). During the present cycle their centers of weight are almost in phase; the $\mathrm{N}-\mathrm{S}$ difference between them is only -0.03 months in the case of $N_{\mathrm{G}}$ and -six months in the case of $N_{\mathrm{F}}$. The value of $N_{\mathrm{GN}}-N_{\mathrm{GS}}$ 

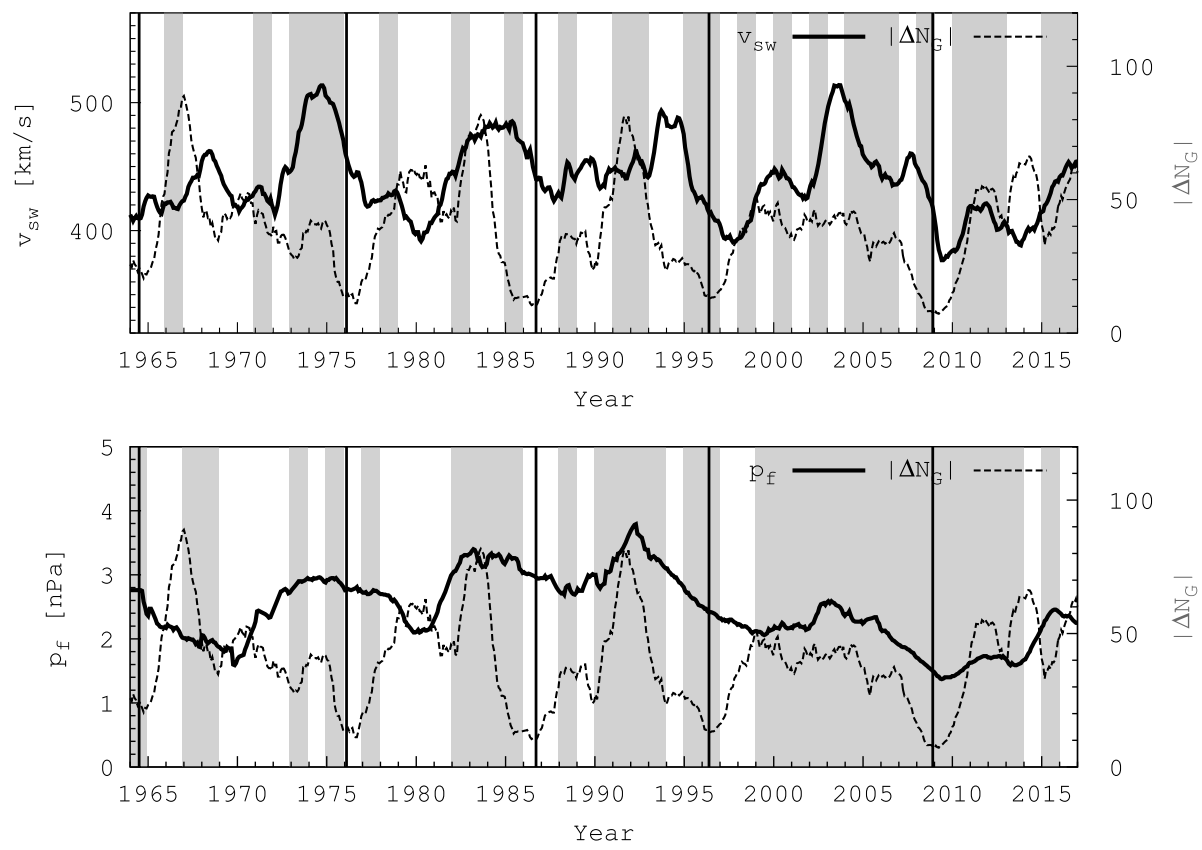

Figure 2 Solar-wind speed (top panel) and flow pressure (bottom panel) with black curves scaled on the left axis versus absolute values of the variations of the difference of hemispheric activity plotted with dashed curves scaled on the right axis. The profiles are smoothed with a 21-month window and the vertical lines indicate the solar-activity minima. The identical and opposite trends in the variations of $\left|\Delta N_{\mathrm{G}}\right|$ and $v_{\mathrm{sw}}$ or $p_{\mathrm{f}}$ are distinguished by gray/white stripes, respectively.

shows that the northern hemisphere leads in time with less than one day and about half a year analyzing the difference between the hemispheric CWs of the flares. Although Cycle 24 is not yet complete, the trend seems to fit the regularity found earlier, because the northern time profile is stronger in the descending phase. If the case will be the same until the next minimum, the northern $\mathrm{CW}$ should move to the right, which means it will get more behind, and thus the time difference between the two hemispheric CWs will be higher and positive. Moreover, the present cycle is similar to Cycle 16, which was also extremely asymmetric. Although its CWs were distinct, the curves of its hemispheric cycles exhibited the same trend as those of Cycle 24 (Muraközy, 2016). Nevertheless, Cycle 24 began with strong northern leading, and now it seems that this cycle may be controlled by the rule of $4+4$ cycles as well (Muraközy and Ludmány, 2012).

After the investigation of the solar-hemispheric flare-production rate, the propagation of the solar wind in the interplanetary space, the flow pressure, and the interplanetary magneticfield strength, as well as the Ap, aa, and the Disturbance Storm-Time [Dst] indices have been compared with the absolute value of the asymmetry of the solar $\mathrm{N}-\mathrm{S}$ hemispheric activity $\left[\left|\Delta N_{\mathrm{G}}\right|\right]$.

For a better comparison of these pairs of curves, the trends of their monthly variations can be examined in the following way. Their relative trend is positive when the two curves change in the same direction during the considered time interval, and it is negative when their relative trend is opposite.

Because their monthly values are stochastic, the characteristic trend of the variations can be described by the yearly mean of their monthly values. The identical and opposite trends 


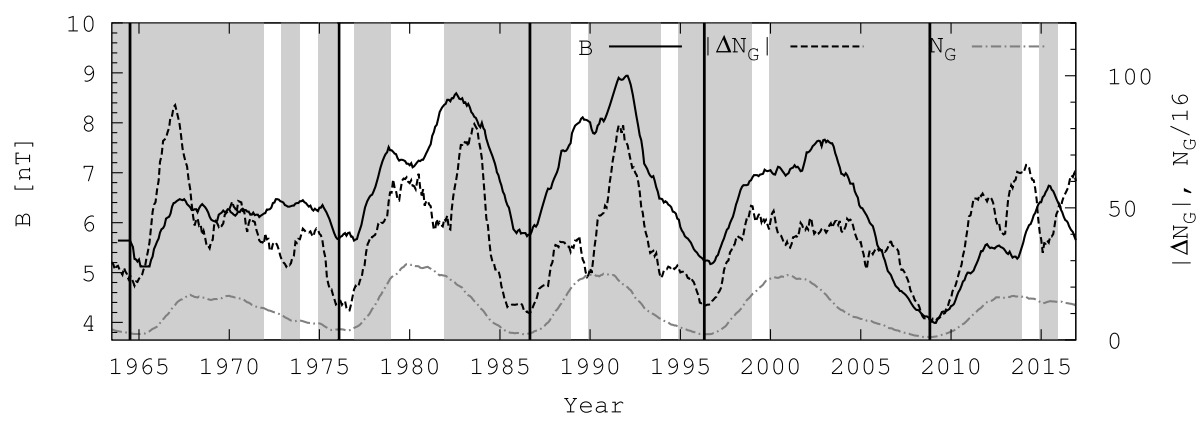

Figure 3 Relation between variations of the magnetic field denoted by the solid black line scaled on the left axis and the $\left|\Delta N_{\mathrm{G}}\right|$ by the dashed black line scaled on the right axis. The dotted gray curve depicts the shape of the total $N_{\mathrm{G}}$ (which is divided by 16 to fit to the same axis). The profiles are smoothed with a 21-month window and the vertical lines indicate the solar-activity minima. The identical and opposite trends in the variations of $\left|\Delta N_{\mathrm{G}}\right|$ and IMF are distinguished by gray/white stripes, respectively.

mentioned above are indicated in the background of the next three figures with gray and white stripes, respectively.

No relationship can be found between $v_{\mathrm{sw}}$ and $\left|\Delta N_{\mathrm{G}}\right|$ in the top panel of Figure 2. In this case the lengths of the positive and negative relative trends are almost the same: $48 \%$ and $52 \%$, respectively. As the bottom panel of Figure 2 shows, a relatively strong relationship can be recognized between the variation of the flow pressure and the asymmetry of solarhemispheric activity. The rate of the positive relative trend is $64 \%$.

Figure 3 shows the relationship between the variations of the $\left|\Delta N_{\mathrm{G}}\right|$ and the magneticfield strength $[B]$ of the interplanetary field. In this figure one can see that the shape of the $\left|\Delta N_{\mathrm{G}}\right|$-curve resembles the shape of the $B$-curve; their peaks are close to each other.

However, the similarity of the $B$-curve to the curve of total sunspot activity $\left[N_{\mathrm{G}}\right]$ depicted by the dotted gray line, is weaker. This is unexpected. The relative trend between $B$ and $\left|\Delta N_{\mathrm{G}}\right|$ is positive during $80 \%$ (45 years) of the investigated time span and in $20 \%$ of the cases is negative. This means that the interplanetary magnetic-field strength may have a relationship with the strength of the solar-hemispheric asymmetry and not merely with the solar activity.

In the cases of the Ap in the top panel and the Dst-index in the bottom panel of Figure 4 one can see that the trend of the variation of $\left|\Delta N_{\mathrm{G}}\right|$ is similar to the curves of the planetary indices studied. The scale of the Dst-index is reversed because the stronger the magnetic disturbance, the lower the value of Dst. The two pairs of curves have local extrema more or less at the same time. The most conspicuous phenomenon in Figure 4 is that the Apand the Dst-indices have extreme minima near the times of the changes in the $4+4$ cycle phase-lag variations marked with vertical solid black lines near 1964 and 2008 (Muraközy and Ludmány, 2012). The relative trend between Ap and $\left|\Delta N_{\mathrm{G}}\right|$ is positive during $64 \%$ of the investigated time span, while the ratio is $65 \%$ in the case of Dst and $\left|\Delta N_{\mathrm{G}}\right|$.

Because the aa-index was introduced to monitor geomagnetic activity over the longest possible time period, one can check whether this trend holds since the beginning of GPR (Figure 5). In the case of aa, the trend is similar to the case of the Ap-index. The relative trend between the aa-index and $\left|\Delta N_{\mathrm{G}}\right|$ is positive during $63 \%$ of the investigated longer time span. The aa-index also has extreme minima near the times of the changes in the $4+4$ cycle phase lags variations near 1964 and 1923 (Muraközy and Ludmány, 2012), but this cannot be pinpointed on the first four cycles of the GPR. 

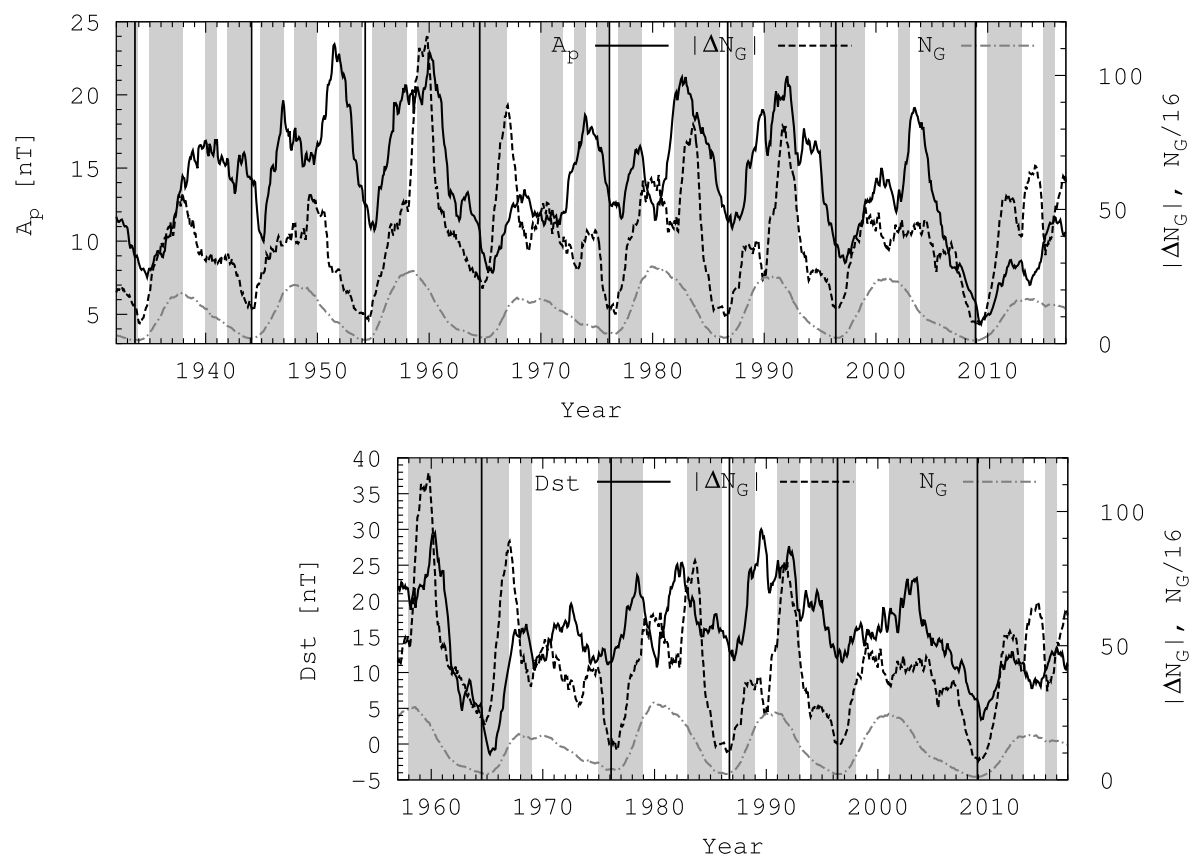

Figure 4 Relation between the variations of the absolute value of the difference between the solar-hemispheric sunspot-group number (dashed black curves scaled on the right axes) and Ap (top panel) as well as the reversed scaled Dst-indices (bottom panel) plotted with solid curves scaled on the right axis. The dotted gray curve depicts the shape of the total $N_{\mathrm{G}}$ (which is divided by 16 to fit on the same axis). The profiles are smoothed with a 21-month window and the vertical lines indicate the solar-activity minima. The identical and opposite trends in the variations of $\left|\Delta N_{\mathrm{G}}\right|$ and the Ap- or Dst-indices are distinguished by gray/white stripes, respectively.

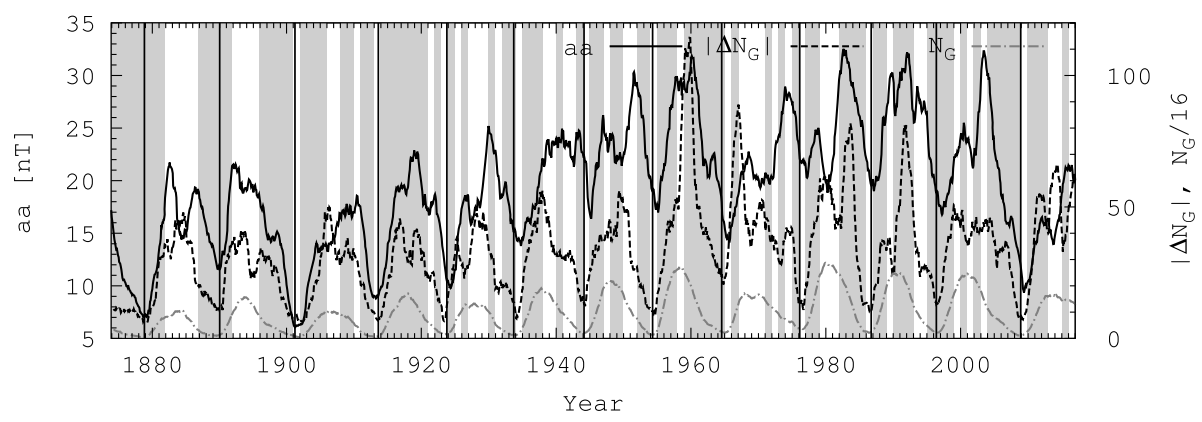

Figure 5 Relation between the variations of the absolute value of the difference between the solar-hemispheric sunspot-group number (dashed black curves scaled on the right axes) and aa index plotted with solid curve scaled on the left axis. The dotted gray curve depicts the shape of the total $N_{\mathrm{G}}$ (which is divided by 16 to fit on the same axis). The profiles are smoothed with a 21-month window and the vertical lines indicate the solar-activity minima. The identical and opposite trends in the variations of $\left|\Delta N_{\mathrm{G}}\right|$ and the aa-index are distinguished by gray/white stripes, respectively. 
The most intriguing result of the present work is that the variations of the interplanetary magnetic field and the geomagnetic indices seem to be more closely related to the variation of the asymmetry of the solar-hemispheric sunspot activity than to the variation of the global sunspot activity. The cause of this behavior is still unclear. This requires more detailed and extended studies by taking into consideration other geomagnetic indices as well.

Acknowledgments J. Muraközy is thankful for the World Data Center for Geomagnetism, Kyoto, and its data suppliers as well as the Hungarian Academy of Sciences (INKP2017/2) and UN Office for Outer Space Affairs travel grants that made possible her participation on the UN/US International Space Weather Initiative Workshop held in Boston, MA, USA, 2017. The author is deeply indebted to the anonymous reviewer for the improvement of the manuscript as well as ideas and encouragement for continuing this study. Open access funding provided by MTA Research Centre for Astronomy and Earth Sciences (MTA CSFK).

Disclosure of Potential Conflicts of Interest The author declares that she has no conflicts of interest.

Publisher's Note Springer Nature remains neutral with regard to jurisdictional claims in published maps and institutional affiliations.

Open Access This article is distributed under the terms of the Creative Commons Attribution 4.0 International License (http://creativecommons.org/licenses/by/4.0/), which permits unrestricted use, distribution, and reproduction in any medium, provided you give appropriate credit to the original author(s) and the source, provide a link to the Creative Commons license, and indicate if changes were made.

\section{References}

Baranyi, T., Győri, L., Ludmány, A.: 2016, Solar Phys. 291, 3081. ADS. DOI.

Georgieva, K., Kirov, B., Javaraiah, J., Krasteva, R.: 2005, Planet. Space Sci. 53, 197. ADS. DOI.

Győri, L., Baranyi, T., Ludmány, A.: 2017, Mon. Not. Roy. Astron. Soc. 465, 1259. ADS. DOI.

Muraközy, J.: 2016, Astrophys. J. 826, 145. ADS. DOI.

Muraközy, J., Ludmány, A.: 2012, Mon. Not. Roy. Astron. Soc. 419, 3624. ADS. DOI.

Mursula, K., Hiltula, T.: 2003, Geophys. Res. Lett. 30, 2135. ADS. DOI.

Mursula, K., Hiltula, T., Zieger, B.: 2002, ESA SP 506, 29. ADS.

Rangarajan, G.K., Iyemori, T.: 1998, J. Atmos. Solar-Terr. Phys. 60, 1017. ADS. DOI.

Virtanen, I., Mursula, K.: 2010, J. Geophys. Res. 115, A09110. ADS. DOI.

Wang, Y.-M., Robbrecht, E.: 2011, Astrophys. J. 736, 136. ADS. DOI.

Zhang, L., Mursula, K., Usoskin, I.: 2013, Astron. Astrophys. 552, A84. ADS. DOI.

Zieger, B., Mursula, K.: 1998, Geophys. Res. Lett. 25, 841. ADS. DOI.

Zolotova, N.V., Ponyavin, D.I.: 2006, Astron. Astrophys. 449, L1. ADS. DOI.

Zolotova, N.V., Ponyavin, D.I.: 2007, Solar Phys. 243, 193. ADS. DOI. 